\title{
CORRESPONDENCE
}

\section{Sceptics and deniers of climate change not to be confused}

Climate-change denial could have disastrous consequences, if it delays global action to cut carbon emissions. Denialism is gaining popularity because people have difficulty differentiating deniers' twisted arguments from the legitimate concerns of genuine sceptics. We must stop deniers presenting themselves as the rightful regulators of scientific debate.

Denial of the science of climate change is eroding public understanding of the issue and seems to be undermining trust in scientists (see, for example, Nature 463, 284-287; 2010). This loss of public confidence after a cold winter in Europe and elsewhere, and the 'Climategate' e-mails controversy - was highlighted at February's meeting of the American Association for the Advancement of Science in San Diego, California (R. J. Cicerone Science 327, 624; 2010).

Denialism is motivated by conviction rather than evidence. It has been applied to a wide range of issues, including evolution and the link between HIV and AIDS. Deniers use strategies that invoke conspiracies, quote fake experts, denigrate genuine experts, deploy evidence selectively and create impossible expectations of what research can deliver. They rely on misrepresentation and flawed logic (P. Diethelm and M. McKee Eur. J. Public Health 19, 2-4; 2009).

By contrast, scepticism starts with an open mind, weighs evidence objectively and demands convincing evidence before accepting any claim. It contributes to the debate and forms the intellectual cornerstone of scientific enquiry.

The public should understand the difference between deniers and sceptics, so that their trust in scientists is not threatened at a time when humanity needs us most. We need to expose the spurious nature of denialist arguments and draw attention back to the primary evidence.

As scientists, we have a duty to communicate our research honestly and accessibly. We do not need to speak with one voice about climate change, but we should stand together to defend proper scientific debate.

Jeremy Kemp Business School, University of Edinburgh, Bristo Square, Edinburgh EH8 9AL, UK

e-mail: j.m.kemp@sms.ed.ac.uk Richard Milne School of Biological Sciences, University of Edinburgh, Edinburgh, UK

Dave S. Reay School of GeoSciences, University of Edinburgh, Edinburgh, UK

\section{Fishermen contribute to protection of marine reserves}

Fisheries benefit from protected marine areas, as eggs, larvae and adult fish spill over into adjacent fishing grounds. But reserves should benefit fishermen too (Nature 463, 1007; 2010). This encourages their compliance, which is essential for fisheries' success.

The real-world fight of fishermen against fishing bans increases the uncertainty in fisheries modelling. Change will come only once fishermen are more involved in conservation and sustainable practices, for example by helping in management experiments or in setting up protected areas under the guidance of scientists.

This has been done successfully in the Torre Guaceto marine reserve in Italy - a country where enforcement and compliance are often weak. Fishermen were involved from the outset when the reserve's management changed five years ago. They participated in refining the management protocol to respect their own traditions and customs, and in monitoring results and decision-making.

When part of the reserve was opened to fishing, they soon saw their income start to increase. They willingly tailored their fishing to comply with agreements reached by marine ecologists and managers on the basis of scientific data. Yields have consistently been roughly double those from fishing grounds outside the reserve (P.G. and J.C. Conserv. Biol. 24, 312-318; 2010).

This success has boosted the trust between fishermen and scientists. The fishermen feel responsible for managing 'their' stock and for enforcing the co-managed framework. Policymakers now aim to extend this co-management approach to the country's other marine reserves.

Joachim Claudet, Paolo Guidetti Laboratory of Zoology and Marine Biology, Department of Biological and Environmental Science and Technologies, University of Salento, CoNISMa, via Monteroni, 73100 Lecce, Italy

e-mail: joachim.claudet@gmail.com

\section{Public database for HIV drug resistance in southern Africa}

The Opinion article by S. Karim and Q. Karim laments the lack of an effective conduit between South Africa's AIDS research and its prevention and treatment policies and programmes (Nature 463, 733-734; 2010). We would like to draw attention to an HIV-1 drug-resistance database, a scientific resource for regional and global HIV research that will enhance surveillance programmes in southern Africa.

The database was established by investigators from the Southern African Treatment and Resistance Network (SATuRN), in collaboration with researchers from the United States and Europe. SATuRN will provide national departments of health with highquality, up-to-date information to guide delivery of antiretroviral therapy, helping to ensure the long-term success of antiretroviral treatment programmes.

As part of this network, we have installed a South African mirror of the Stanford HIV Drug Resistance
Database (HIVDB). This mirror (www.bioafrica.net/saturn) will be continuously updated and released to local investigators in a curated and readily analysable form, in the context of more than 120,000 sequences already in the Stanford HIVDB. Neighbouring countries that share subtype $C$ as the predominant virus (the strain fuelling southern Africa's AIDS epidemic) are also providing data.

The mirror will ensure that subtype- $C$ sequences are analysed according to standard state-of-the-art technologies developed by Stanford HIVDB. It will help patient management, allowing quick identification of resistant strains and systematic tracking both of resistance patterns and of prevalence and distribution of resistance mutations within different population groups. It will inform decisions about new drugs, diagnostics and treatment strategies in southern Africa. Already, the data show that resistance in newly infected individuals is still very low (under $5 \%$ ), as is the accumulation of thymidine-analogue mutations that can limit the effectiveness of second-line antiretroviral therapy.

Investigators, clinicians and laboratories wishing to take part in the collaboration should contact the authors.

Tulio de Oliveira Africa Centre for Health and Population Studies, Nelson R. Mandela School of Medicine, University of KwaZulu-Natal, Durban,

South Africa

e-mail: tdeoliveira@africacentre.ac.za Robert W. Shafer Division of Infectious Diseases, Department of Medicine,

Stanford University, USA

Christopher Seebregts Biomedical Informatics Research, Medical

Research Council, South Africa, and others (full author list at go.nature. com/9VaZsP)

Contributions may be submitted to correspondence@nature. com. Please refer to the Guide to Authors at http://go.nature.com/ cMCHno. Published contributions are edited. Readers are welcome to comment online. 